Bio-grafía. Escritos sobre la Biología y su Enseñanza. ISSN 2027-1034

Número Extraordinario. p.p. 162-171

Memorias del Primer encuentro ambiental Universidad, ambiente y sustentabilidad: experiencias y prácticas.

\title{
PATOLOGÍAS CAUSADAS POR EXPOSICIÓN AL AMIANTO.
}

\author{
Acosta Barreto Luz Angela ${ }^{1}$ \\ Carreño Angie Katherine ${ }^{1}$ \\ Rendón Rojas Ginneth Lorena ${ }^{1}$
}

\section{Resumen}

La presente investigación pretende dar a conocer la influencia que tiene el amianto en el desarrollo de patologías pulmonares. La revisión de la literatura, pone en evidencia una fuerte vinculación entre este mineral y enfermedades como el mesotelioma, cáncer de pulmón y asbestosis; que se producen en personas que trabajaron en la industria del asbesto, razón por la cual tuvieron una exposición directa a éste mineral. Sin embargo, sorprende que tanto la revisión bibliográfica, como la de casos ponen de manifiesto que esta fibra resulta ser perjudicial para otros individuos que a pesar de no estar expuestos directamente, si inhalan en su momento éstas peligrosas fibras. (Escobar, 2012)

Por otra parte, se pretende abordar cada una de las patologías relacionadas con el amianto explicando el comportamiento de la fibra dentro del organismo, el daño que este causa y su respectivo tratamiento.

Palabras clave: Amianto, mineral, vías respiratorias, asbestosis, cáncer

\section{Abstract}

The present investigation will announce the concept of this mineral; his reasons and consequences. The direct entail of this mineral with diseases like the mesothelioma and cancer of lung on having been exposed every day in the different industries recognized in Colombia. On the other hand the behavior of the fiber inside the organism in each of the related pathologies direct and indirectly with the asbestos; the hurt that this one causes and his respective treatment.

Keywords: Asbestos, mineral, respiratory tract, asbestosis, cancer.

Universidad Pedagógica Nacional, Estudiante de Licenciatura en Química, SISMA Semillero de investigación en salud y medio ambiente. Email: luzab4@gmail.com 
Bio-grafía. Escritos sobre la Biología y su Enseñanza. ISSN 2027-1034

Número Extraordinario. p.p. 162-171

Memorias del Primer encuentro ambiental Universidad, ambiente y sustentabilidad: experiencias y prácticas.

\section{INTRODUCCIÓN}

El asbesto (amianto) es un mineral con fibras flexibles, brillantes suaves y con gran resistencia a altas temperaturas. (Rivera, 2013).

La inhalación de estas fibras se considera de alto riesgo para la salud. Puesto que sus características particulares (tamaño pequeño, baja densidad y forma delgada y larga) influyen en su penetración a las vías respiratorias y el consecuente desarrollo patológico. (Rivera, 2013)Las probabilidades de que esta fibra sea inhalada son muy altas, dado que éstas pueden permanecer en suspensión durante mucho tiempo.

Aunque no todas las personas expuestas al mineral contraerán una enfermedad relacionada con este, todas tienen un alto riesgo de contraer alguna patología derivada del asbesto; a menos que ellas no se prevengan, el índice de mortalidad a causa de la inhalación de esta fibra seguirá creciendo, puesto que dichas afecciones son difíciles de tratar y su mayoría no tiene un tratamiento de curación efectiva.

Algunos testimonios, indagación en las zonas de prevalencia de las patologías, así como diferentes investigaciones y la certeza que la legislación de más de 60 países prohíben su uso; comprueban que algunas patologías como la asbestosis, mesotelioma maligno y cáncer de pulmón, son producidas por este mineral. (Regueiro, 2008)

La patogénesis de éste tipo de enfermedades reviste una ruta común, que comienza por el contacto directo o indirecto con la fibra, la inhalación de la misma y su penetración en las estructuras del sistema respiratorio. Una vez dentro de los pulmones, se da paso a la activación del sistema inmune quien da inicio a los mecanismos de defensa del organismo, dando paso a procesos inflamatorios y una serie de reacciones químicas que pretenden ubicarlas, descomponerlas y expulsarlas.

Sin embargo, muchas de las fibras de amianto consiguen quedarse en el cuerpo durante mucho tiempo causando graves lesiones en el individuo que actúa como portador, quien generalmente adolece de síntomas asociados, haciendo que el diagnóstico y tratamiento no puedan ser implementados en fases tempranas del desarrollo patológico. Es así como la prevalencia de la enfermedad se presenta a muy largo plazo, generalmente después de 20 años. (Carmona, 2012) 
Bio-grafía. Escritos sobre la Biología y su Enseñanza. ISSN 2027-1034

Número Extraordinario. p.p. $162-171$

Memorias del Primer encuentro ambiental Universidad, ambiente y sustentabilidad: experiencias y prácticas.

Hasta la fecha, no se ha encontrado un tratamiento eficaz para las enfermedades que produce el asbesto, aun cuando se evidencia que el daño que éste causa en el organismo incluso puede llegar a la muerte.

Por ello, el manejo preventivo de esta fibra está relacionada con la higiene en los diferentes lugares de trabajo y así mismo con la protección del trabajador (tapabocas industrial, overoles especiales, uso adecuado del material, ventilación, etc.); en el caso de la demolición de una estructura o edificio se debe tener en cuenta qué tipo de material está construido, para que al momento de su devastación, no queden fibras de este mineral en suspensión.

Desde la parte médica se debe realizar un control o seguimiento médico detectando a tiempo la patología; estos controles se llevan a cabo periódicamente (exploración del sistema respiratorio) obteniendo un historial médico de cada trabajador; sin embargo, la "única prevención" real para la asbestosis pleuro-pulmonar y el mesotelioma pleural maligno se daría mediante políticas públicas que privilegien la vida y la integridad humana por encima de otros intereses. Esto conlleva, sin lugar a duda a la prohibición de la utilización de éste material dentro de las industrias relacionadas con el asbesto.

Cuando la enfermedad es descubierta se pueden llevar a cabo ciertos tratamientos como lo son:

Quimioterapia: se realizan diferentes esquemas terapéuticos utilizando como medicamento base el platino que van acompañados de pemetrexed, la gemcitabina, la doxorrubicina o la vinorrelbina. (Carmona, 2012)

Cirugía: la pleurectomía consiste en extirpar las dos membranas pleurales, la parietal que es la que recubre la caja torácica y la visceral que recubre los pulmones con el objetivo de disminuir la masa del tumor.

Radioterapia: tiene como objetivo disminuir el tumor colocando una sonda de drenaje.

Tratamiento multimodal: tiene un objetivo de combinar los diferentes tratamientos para extender el tiempo de vida del paciente; además se realiza drenajes y la percusión del tórax. 
Bio-grafía. Escritos sobre la Biología y su Enseñanza. ISSN 2027-1034

Número Extraordinario. p.p. 162-171

Memorias del Primer encuentro ambiental Universidad, ambiente y sustentabilidad: experiencias y prácticas.

\section{MARCO TEÓRICO}

\section{Enfermedades relacionadas con el Asbesto.}

El asbesto es una de las principales causas de tumores malignos presentes en el organismo de personas que trabajan con exposición directa al polvo de amianto (minas de extracción del mineral, fábricas de material de construcción, embragues o frenos de carros, algunos textiles y fabricación de trajes de aproximación al fuego). (Rivera, 2013) No obstante, estas fibras también pueden estar al interior de los hogares, pues el amianto es un mineral muy utilizado como materia prima en productos que son empleados diariamente por la población colombiana. (Escobar, 2007)

Por otra parte, la revisión de la literatura disponible permite establecer una relación directa entre enfermedades como mesotelioma y cáncer de pulmón producidas por la exposición a este minera (Reguerio, 2008); mientras se afirma que enfermedades como EPOC y neoplasias de ovarios, laringe y riñón no son directamente causadas por el amianto. (Carmona, 2012) Sin embargo, se puede afirmar que si la exposición continúa puede aumentar el riesgo individual que conlleva en muchos de los casos al desarrollo de las patologías mencionadas. (Martinez, 2005)

Es así como se pueden clasificar las enfermedades producidas por esta fibra mortal, en leves (benignas) y peligrosas (malignas); siendo, éstas últimas, las menos frecuentes. Sin embargo, llama la atención que algunas de las enfermedades clasificadas como benignas (ejemplo la asbestosis) son tan graves como aquellas consideradas con un riesgo mayor (tal es el caso del cáncer), dada la tasa de mortalidad tan alta que presentan.

Para conocer un poco más a profundidad las patologías en estudio, se presentarán definiciones y características asociadas a continuación:

\section{Mesotelioma}

El tejido que recubre los pulmones, el estómago, el corazón y otros órganos se llama mesotelio; esta enfermedad es relacionada con un tumor de este tejido. El mesotelioma pleural es el responsable del 75 por ciento de los diagnósticos relacionados con el asbesto.

Las fibras de este mineral, viajan a la membrana pleural después de ser inhaladas y es allí donde se alojan. Al pasar el tiempo, termina por irritar la pleura generando procesos inflamatorios que desencadenan la consabida 
Bio-grafía. Escritos sobre la Biología y su Enseñanza. ISSN 2027-1034

Número Extraordinario. p.p. $162-171$

Memorias del Primer encuentro ambiental Universidad, ambiente y sustentabilidad: experiencias y prácticas.

neoplasia. Ello porque el asbesto ingresa a las células pleurales y si estas son cancerígenas se multiplican formando tumores. (Reguerio, 2008)

Cabe aclarar que el pulmón posee dos capas que lo recubren, la externa se denomina pleura parietal y la interna es conocida como capa visceral. El mesotelioma se desarrolla en la capa visceral de la pleura e invade lugares cercanos como el diafragma, las paredes del pecho o el pulmón.

Este cáncer también puede aparecer en el revestimiento del estómago, el corazón o los testículos generando patologías que se conocen como mesotelioma peritoneal, pericárdico y/o testicular. El diagnóstico puede presentar diferentes síntomas pero el dolor de pecho o dolor abdominal y falta de aire afecta a la mayoría de los pacientes, independientemente del cáncer que presente. (Dueñas, 2001)

Síntomas:

Es posible que estos aparezcan 20 o 40 años después de la exposición al asbesto. Los síntomas que se presentan son:

- Distensión abdominal.

- Dolor abdominal.

- Dolor torácico.

- Tos.

- Fatiga.

- Dificultad respiratoria.

- Pérdida de peso.

- Fiebre y sudoración.

\section{Cáncer de pulmón}

Los pulmones son un par de órganos respiratorios los cuales llevan oxígeno al cuerpo cuando se inhala y expulsan dióxido de carbono cuando se exhala De este modo en la inhalación las fibras de asbesto entran al organismo por las vías respiratorias quedando almacenadas en los pulmones dando lugar al desarrollo de patologías mortales.

El amianto es responsable tan solo de una pequeña parte de todos los diagnósticos de cáncer, y aun así el cáncer de pulmón sigue siendo una de 
Bio-grafía. Escritos sobre la Biología y su Enseñanza. ISSN 2027-1034

Número Extraordinario. p.p. $162-171$

Memorias del Primer encuentro ambiental Universidad, ambiente y sustentabilidad: experiencias y prácticas.

las enfermedades relacionados con este mineral, cuya tasa de morbilidad es muy elevada.

Síntomas:

- Cansancio.

- Pérdida de apetito.

- Tos seca o con flemas.

- Tos con sangre en el esputo.

- Dificultad para respirar (Disnea).

- Dolor.

\section{Cáncer de ovarios}

También se puede identificar cáncer de ovarios por contacto con amianto, dado que el tamaño de las partículas de éste material puede ingresar por los órganos a través del torrente sanguíneo, sistema linfático o el tracto reproductivo.

Este tipo de neoplasia evidencia pocos síntomas y atraviesa un sinnúmero de fases antes de generarlos, lo que imposibilita una detección temprana y un tratamiento pertinente. Así cuando éstos aparecen, generalmente resulta ser tardío. Sin embargo se reconoce que la enfermedad genera como uno de sus síntomas más reconocidos, un leve dolor en el abdomen que después de persistir un largo tiempo avizora una hemorragia uterina. Otro síntoma es que pueda aparecer un líquido en el abdomen, generando una hinchazón, crecimiento excesivo del revestimiento interno uterino y cansancio. (Dueñas, 2001)

\section{Síntomas:}

- Fatiga.

- Tos.

- Falta de aliento.

- Dolor en el tórax.

- Pérdida del apetito.

- Tos con flema o moco.

- Tos con sangre. 
Bio-grafía. Escritos sobre la Biología y su Enseñanza. ISSN 2027-1034

Número Extraordinario. p.p. $162-171$

Memorias del Primer encuentro ambiental Universidad, ambiente y sustentabilidad: experiencias y prácticas.

\section{Cáncer de laringe}

Este tipo de cáncer no es tan común en relación con el asbesto, aunque existe un vínculo entre las fibras y la enfermedad. Otras razones por las que se presenta esta patología están asociadas al tabaquismo o el consumo excesivo de alcohol. Más de 13.500 casos se diagnostican cada año en los EE.UU. y aproximadamente el 60 por ciento de las personas viven más de cinco años. (Dueñas, 2001)

\section{Síntomas:}

- Sonidos respiratorios anormales (sonidos chillones).

- Tos.

- Expectoración con sangre.

- Dificultad en la deglución.

- Ronquera que no mejora en 3 o 4 semanas.

- Dolor en el cuello o en el oído.

\section{Asbestosis}

Es una enfermedad que se genera debido a que las fibras de amianto producen una cicatrización, limitando la respiración e infiriendo en la capacidad de ingreso de oxígeno en el torrente sanguíneo; esta enfermedad también es conocida como fibrosis pulmonar. En la mayoría de los casos este cáncer se genera debido a la exposición del asbesto, por lo general, la asbestosis aparece entre diez y veinte años después del inicio de la exposición a este mineral.

\section{Síntomas:}

- Dolor torácico.

- Tos.

- Dificultad respiratoria con el ejercicio (empeora lentamente con el tiempo).

- Opresión en el pecho.

Otros posibles síntomas incluyen:

- Dedos en palillo de tambor.

- Anomalías ungulares. 
Número Extraordinario. p.p. 162-171

Memorias del Primer encuentro ambiental Universidad, ambiente y sustentabilidad: experiencias y prácticas.

\section{Otros cánceres relacionados}

Otros tipos de cáncer que están estrechamente asociados con la exposición al amianto incluyen el cáncer de esófago, cáncer de vesícula biliar, cáncer de riñón y cáncer de garganta. Algunos estudios confirman casos en los que se relaciona la exposición del mineral con estos tipos de cáncer, pero no es un factor de riesgo comprobado.

\section{¿Cómo sabemos que el asbesto es un riesgo para la salud?}

Varios estudios de entidades como el Departamento de Salud y Servicios Humanos de Estados Unidos clasifican el asbesto como un cancerígeno humano. (Sancho, 2016) Así mismo el Instituto Nacional Del Cáncer nos confirma que el asbesto causa asbestosis, mesotelioma, cáncer de pulmón, y otros tipos de cáncer.

Uno de los estudios más importantes investigó las actas de defunción del sindicato de trabajadores que han tenido contacto con este mineral. Todos habían trabajado con cantidades peligrosas de asbesto durante al menos 20 años. (The Center To Protect Workers' Rights, 2012) Muchas de las personas que laboraban en estas fábricas no tuvieron ningún tipo de prevención, pues no sabían el nivel de riesgo que presentaba manipular esta fibra. Varios de ellos murieron simultáneamente de enfermedades relacionadas con este mineral.

\section{¿Qué tan peligroso es el asbesto?}

Ante el cuestionamiento de si es posible usar el mineral bajo un sistema de uso seguro, el académico Carlos Alberto Agudelo (Sancho, 2016) señala que de acuerdo con la evidencia mundial no hay límite seguro y ya se ha prohibido en más de 56 países.

Según reportó el Ministerio de Trabajo, se han identificado 17 enfermedades laborales relacionadas con el uso de este mineral en múltiples aplicaciones como enchapes, techos, tejas y pastillas de frenos para carros. (The Center To Protect Workers' Rights, 2012)

Todas las enfermedades producidas por el asbesto (con excepción de una) están relacionadas con la dosis de exposición, es decir que mientras más expuesto esté a las fibras de amianto, tendrá más posibilidades de que estas fibras entren a su sistema respiratorio y así mismo se desarrolle una de estas enfermedades. La única enfermedad causada por el asbesto que es 
Número Extraordinario. p.p. 162-171

Memorias del Primer encuentro ambiental Universidad, ambiente y sustentabilidad: experiencias y prácticas.

un poco diferente es el mesotelioma, que se puede contraer incluso con cantidades muy pequeñas de asbesto. Algunas familias de trabajadores expuestos a la fibra del mineral han sido propensos a contraer una de las enfermedades relacionadas con el asbesto, puesto que algunas empresas que manejan el mineral como materia prima no tienen un buen manejo de protección personal dejando así que los trabajadores lleven la ropa con la que manipulan el mineral a sus casas y dejando así a sus familias expuestas a esta mortal fibra.).

\section{¿Cuánto tiempo lleva contraer enfermedades causadas por el asbesto?}

Todas las enfermedades causadas por el asbesto tienen un periodo de latencia, que es el periodo que transcurre entre el momento en que estuvo expuesto al asbesto y el momento en que comienza a sentirse enfermo. El periodo de latencia para las enfermedades derivadas del asbesto dura entre diez y cuarenta años. Puede enfermarse entre diez y cuarenta años después incluso si sólo trabajó con asbesto por un año y luego dejó de hacerlo. (Rivera, 2013).

\section{MATERIALES Y MÉTODOS}

Este tipo de investigación posee un enfoque estático es decir de tipo no experimental; apoyada principalmente por revisión documental.

\section{CONCLUSIONES Y RECOMENDACIONES}

Los datos reportados mediante este artículo suponen argumentos científicos que constituyen bases iniciales para comprender el impacto del asbesto en la salud humana y sus implicaciones frente a la exposición que puede llegar a darse en comunidades aledañas a fábricas y/o centros de explotación donde se utilice dicho mineral. Aunque ya se hizo un primer esfuerzo por compartir la información recolectada e indagar en la percepción que tienen los habitantes del barrio Pablo Neruda ubicado en vía Sibaté, frente a la problemática del asbesto no contamos con el nivel de información y molestia que esperábamos en sus pobladores. Sin embargo, se hizo evidente que todo el pueblo está expuesto a esta fibras y que este sitio fue construido sobre grandes cantidades de residuos que eran desechadas por una de las industrias más reconocidas en Colombia (fabrica Eternit), que emplea como insumo el asbesto para la fabricación de esos productos, lo que impacta directamente en el estado de salud de los habitantes y posiblemente genera algunas de las enfermedades descritas. 
Bio-grafía. Escritos sobre la Biología y su Enseñanza. ISSN 2027-1034

Número Extraordinario. p.p. $162-171$

Memorias del Primer encuentro ambiental Universidad, ambiente y sustentabilidad: experiencias y prácticas.

Se hace necesario implementar un acompañamiento a la población residente, mediante el cual se indique el impacto que genera el asbesto y como puede ser mitigado. Además de hacer pedagogía en torno a la necesidad de prohibir su uso como único método eficaz de control.

\section{BIBLIOGRAFÍA}

https://www.asbestos.com/mesothelioma/related-diseases.php

http://www.amianto.info/que_es_el_amianto.html

https://espanol.asbestos.com/mesotelioma/pleural.php

https://medlineplus.gov/spanish/ency/article/000118.htm

http://www.amianto.info/amianto.html

http://www.cpwr.com/sites/default/files/training/asbestos/02\%20ASB\%202rev

ES.pdf

http://agenciadenoticias.unal.edu.co/detalle/article/no-hay-sistema-de-uso-

seguro-para-el-asbesto.html

https://www.cancer.gov/espanol/cancer/causas-

prevencion/riesgo/sustancias/asbesto/hoja-informativa-asbesto\#q3

http://www.scielo.org.mx/pdf/facmed/v56n2/v56n2a2.pdfhttp://www.ladep.es/f icheros/documentos/Normativa\%20sobre\%20el\%20asbesto\%20y\%20sus\%2 Oenfermedades\%20pleuropulmonares.\%20Grupo\%20de\%20trabajo\%20ER OL-SEPAR.\%20Arch\%20Bronconeumol.p 